\title{
Adaptive noise cancellation for eliminating artifacts of life signals using fuzzy and neural networks
}

\author{
Mohammad Seifi", Aliakbar Kargaran Erdechi, Ahmad Hajipour \\ Department of Electrical and Computer Engineering, Hakim Sabzevari University, Sabzevar, Iran \\ Email address: \\ mohamad_saifi@yahoo.com (M. Saifi), aliakbar.kargaran@gmail.com (A. kargaran), a.hajipour@hsu.ac.ir (A. Hajipour)
}

\section{To cite this article:}

Mohammad Seifi, Aliakbar Kargaran Erdechi, Ahmad Hajipour. Adaptive Noise Cancellation for Eliminating Artifacts of Life Signals Using Fuzzy and Neural Networks. Journal of Electrical and Electronic Engineering. Special Issue: Research and Practices in Electrical and Electronic Engineering in Developing Countries. Vol. 3, No. 2-1, 2015, pp. 21-24. doi: 10.11648/j.jeee.s.2015030201.15

\begin{abstract}
Electroencephalogram (EEG) is one of the commonly used non-invasive techniques for understanding the brain functions. This paper presents a method for removing electroocular (EOG) artifacts in the electroencephalogram (EEG). A new adaptive radial-basis function- networks- (RBFN-) for the adaptive noise cancellation (ANC) problem is proposed. Also, the algorithm of structure identification and parameters adjustment is developed. The proposed RBFN approach implements Takagi-Sugeno-Kang (TSK) fuzzy systems, functionally. Simulation results demonstrate that the proposed adaptive RBFN can remove the noise successfully and efficiently with a parsimonious structure.
\end{abstract}

Keywords: RBFN, ANC, Adaptive Filtering, Neural Network

\section{Introduction}

Electroencephalogram (EEG) is one of the commonly used non-invasive techniques for understanding the brain functions. As EEG reflects the mental activity, it can also be used as a communication tool for people who cannot control their environment through normal neuromuscular channel. This new mode of communication involves direct connection with brain and computer and development of algorithms to translate features extracted from EEG to commands which are employed to control some output devices in real time [1].

But Eye movements and blinks produce electrical potentials that propagate over the scalp creating significant electrooculographic artifacts in the recorded electroencephalogram. These artifacts often complicate the interpretation of the EEG. Thus potentials created from noncerebral origins may degrade the performance of Brain Computer Interface (BCI). It is very important to ensure that electric signals caused by eye are removed from EEG as they are the most important sources of contamination in BCI systems [2]. The eye is equivalent to an electric dipole with cornea and retina as positive and negative polarity, respectively. When the eye moves, electric field around it changes and it produces an electric signal called electrooculogram (EOG). It propagates over the scalp and appears in the EEG as noise or artifact. Normally EOG signals are of high amplitude, low frequency and affect the lower band power of EEG. The simplest linear filtering method cannot be applied here as frequencies of artifacts and neurological phenomena in EEG overlap. So artifacts are serious problems in the interpretation and analysis of EEG [2]. There are many methods to avoid ocular artifacts from EEG such as Independent Component analysis (ICA) [4], principal component analysis [7], time and frequency domain regression methods [8,9] and adaptive filtering [3]. Among these methods, adaptive filtering gives better results and can be applied in real time $[3,4]$. From $[5,6]$, it can be concluded that the adaptive filtering algorithm using REOG as the third reference input can remove the artifacts more efficiently. In this paper modification of adaptive filtering algorithm is done by using two reference inputs.

\section{Adaptive Noise Cancellation}

The basic method used in this paper for elimination of artifacts is Adaptive Noise Cancellation (ANC). It is a process by which the interference signal can be filtered out by identifying a model between a measurable noise source and the corresponding immeasurable interference. The method uses a noisy signal as primary input and a reference 
input that consists of noise correlated in some unknown way with the primary noise. It is also assumed that the desired clean signal is uncorrelated with noise source and interference signals. By adaptively filtering and subtracting the reference input from the primary input, the output of the adaptive filter will be the error signal, which acts as a feedback to the adaptive filter. With this setup, the adaptive filter will be able to cancel the noise and obtain an estimate of the less noisy signal [6].

Figure 1 shows the basic concept of adaptive noise cancellation using the proposed adaptive RBFN filter The primary input of the canceler is the measurable EEG signal $q(k)$, which is naturally contaminated by artifacts. In other words, the artifact signal $n(k)$ (noise source signal) goes through unknown nonlinear dynamics $f$ (the route in human body from the artifact generating source to each EEG electrode on the scalp) and generates a distorted noise $d(k)$, which is then added to clean EEG signal $s(k)$, in the place of electrodes to form $q(k)=s(k)+d(k)$ which is the very signal measured by the EEG electrodes. The reference input to the canceler, which is the input to the adaptive filter in fact, is the noise source signal $\mathrm{n}(\mathrm{k})$, measured directly from the artifact generating origin i.e. EOG for ocular artifacts. The noise $\mathrm{n}(\mathrm{k})$ is filtered to produce $\hat{d}(k)$ which is as close a replica as possible of interference signal, $d(k)$ the distorted and delayed version of $n(k)$. The aim is to retrieve clean EEG, $s(k)$, from the measured EEG signal, $\mathrm{q}(\mathrm{k})$, that is procurable by estimating the $\hat{d}(k)$ using the adaptive filter, which is then subtracted from measured $\mathrm{q}(\mathrm{k})$ to produce the output of the system, $\hat{s}(k)$ that would be close to the required signal $s(k)$. In the noise canceling application, the objective is to produce an error signal (output) that is a best fit in the least squares sense to the signal $s(k)$ This is accomplished by feeding back the system output to the adaptive filter to minimize the error signal until it reaches the value $\hat{s}(k)=$ $s(k)$. As a result, the output of the noise canceler $\hat{s}(k)$ is the estimation of corrected or clean EEG. $\hat{s}(k)=s(k)+d(k)-$ $\widehat{d}(k)$ that is $\hat{s}(k) \cong s(k) \cdot[6]$

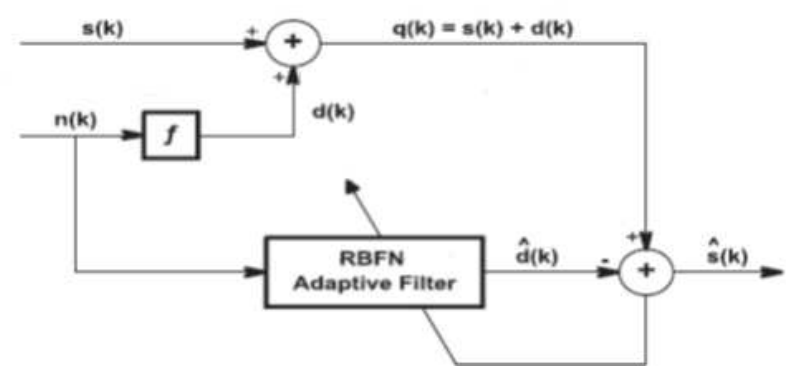

Figure 1. Adaptive Noise Cancellation

\section{Adaptive RBFN-Based Filter}

The adaptive RBFN-based filter to perform TSK inference is depicted in Figure 2. Basically, it is a multi-input singleoutput (MISO) system [10]. It is straightforward to extend the result to multi-input multi-output (MIMO) systems. The functions of the nodes in each of the five layers are described as follows:



Figure 2. Structure of radial-basis function- networks

Layer 1: Each node in layer 1 represents an input node and it corresponds to one input linguistic variable. These nodes simply transmit input signals to the next layer directly.

Layer 2: Nodes in this layer stand for input terms of an input linguistic variable. Each one acts as a one-dimensional membership function (MF) which is a Gaussian function of the following form:

$$
\mu_{i j}\left(\mathrm{x}_{i}\right)=\exp \left(-\frac{\left(\mathrm{x}_{i}-c_{i j}\right)^{2}}{\sigma_{j}^{2}}\right)
$$

Where $i=1,2, \ldots r, \quad r$ is the number of input variables $, \mathrm{j}=1,2, \ldots \mathrm{u}, \mathrm{u}$ is the number of membership (MF) functions,$\mu \mathrm{ij}$, jth membership function of the ith input variable $x i$,cij center of the jth MF of $x i, \sigma j$ width of the jth MF of $x i$

Layer 3: Each node in layer 3 represents a possible IF part of a fuzzy rule. The number of rules in this system is exactly the number of RBF neurons. For the jth rule $\mathrm{Rj}$, its output is

$$
\begin{aligned}
\emptyset_{j}= & \exp \left(-\frac{\sum_{i=1}^{r}\left(\mathrm{x}_{\mathrm{i}}-c_{i j}\right)^{2}}{\sigma_{j}^{2}}\right) \\
= & \exp \left(-\frac{\left\|\mathrm{x}-c_{j}\right\|^{2}}{\sigma_{j}^{2}}\right)
\end{aligned}
$$

where $X=(x 1, \ldots, x r)$ and $C j$ is the center of the $j$ th $R B F$ neuron.

Layer 4: Nodes in this layer are called normalized nodes. The number of normalized nodes is equal to that of RBF neurons. The output of the normalized nodes is given by

$$
\begin{aligned}
\psi_{j} & =\frac{\emptyset_{j}}{\sum_{k=1}^{u} \emptyset_{k}} \\
= & \frac{\exp \left(-\frac{\left\|\mathrm{X}-c_{j}\right\|^{2}}{\sigma_{j}^{2}}\right)}{\sum_{k=1}^{u} \exp \left(-\frac{\left\|\mathrm{X}-c_{k}\right\|^{2}}{\sigma_{k}^{2}}\right)}
\end{aligned}
$$


The nodes in layer 4 are fully connected with the nodes in layer 3 for normalization.

Layer 5: Each node in this layer represents an output variable which is the summation of incoming signals from layer 4. Its output is given by

$$
\begin{aligned}
\mathrm{y}(\mathrm{X}) & =\sum_{k=1}^{u} w_{k} \cdot \psi_{k} \\
= & \frac{\sum_{k=1}^{u} w_{k} \exp \left(-\frac{\left\|\mathrm{X}-c_{k}\right\|^{2}}{\sigma_{k}^{2}}\right)}{\sum_{k=1}^{u} \exp \left(-\frac{\left\|\mathrm{X}-c_{k}\right\|^{2}}{\sigma_{k}^{2}}\right)}
\end{aligned}
$$

where $\mathrm{y}$ is the value of the output variable and $w_{k}$ is essentially the consequent part of the kth fuzzy rule. In the TSK fuzzy inference system, we have

$$
w_{k}=t_{k 0}+t_{k 1} x_{1}+\cdots+t_{k r} x_{r}
$$

where $\mathrm{k}=1,2, \ldots \mathrm{u}$. The parameters $t_{k 0}, t_{k 1}, \ldots t_{k r}$ are the linear parameters in the consequent part of the kth fuzzy rule. From Fig. 2 and the detailed functions of the nodes, it can be seen that the following conditions hold to make the adaptive RBFN-based filter equivalent to a TSK fuzzy inference system.

1) The number of links from layer 2 to each node in layer 3 is equal to the number of input variables. That is, a set of $r$ input term nodes (one for each input linguistic node) in layer 2 is connected to a rule node in layer 3 .

2) Each RBF neuron is a fuzzy rule essentially.

3 ) For the RBF neurons, no bias is considered.

4) The weights between layer 4 and layer 5 are the linear functions instead of a real constant.

\section{Results and Analysis}

EEG data with ocular artifacts are taken from [11] for testing the proposed method. Figure 3 shows the EEG that eliminated with EOG signal and then we applied this signal to the proposed filter.

Figure 3 indicates the performance of the proposed filter in eliminating the ocular artifact. Figure3.a and Figure3.b show the measured EEG signal and directly measured EOG signal, respectively, the estimated ocular interference signal using the proposed adaptive filter is shown in Figure 3.c, and ultimately Figure 3.d illustrates the clean EEG resulting from subtracting the estimated interference signal from the measured EEG as shown in the ANC system. Considering the part marked by arrows that indicate the severe presence of ocular artifacts, it is obvious that the proposed filter has removed the artifacts successfully and has desirable performance in this area. The result is also achieved in frequency domain using power spectrum magnitude (PSM) criterion. Figure 4.a shows the power spectrum of the EEG signal contaminated with EOG, the power spectrum of EOG signal is shown in Figure 4.b and the power spectrum of cleaned EEG is illustrated in Figure 4.c. Note that the absence of low frequencies of EOG in output signal indicates the removal of EOG artifact.
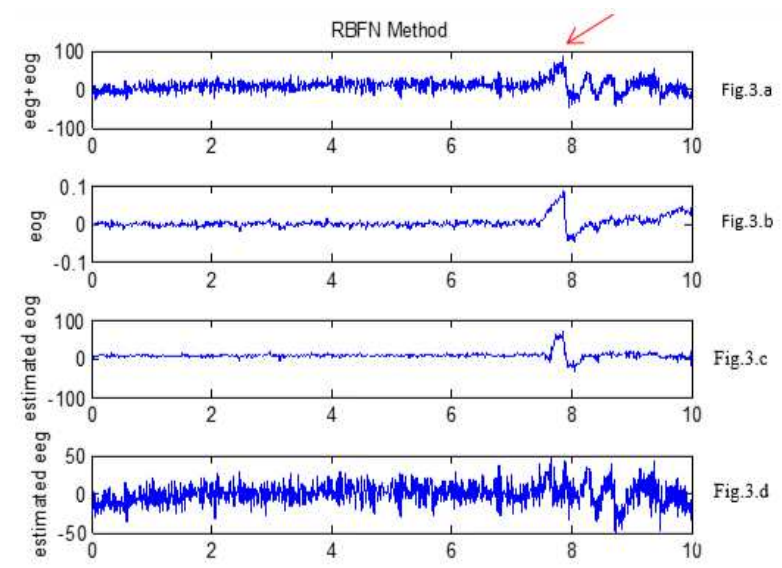

Figure 3. EOG artifact cancellation
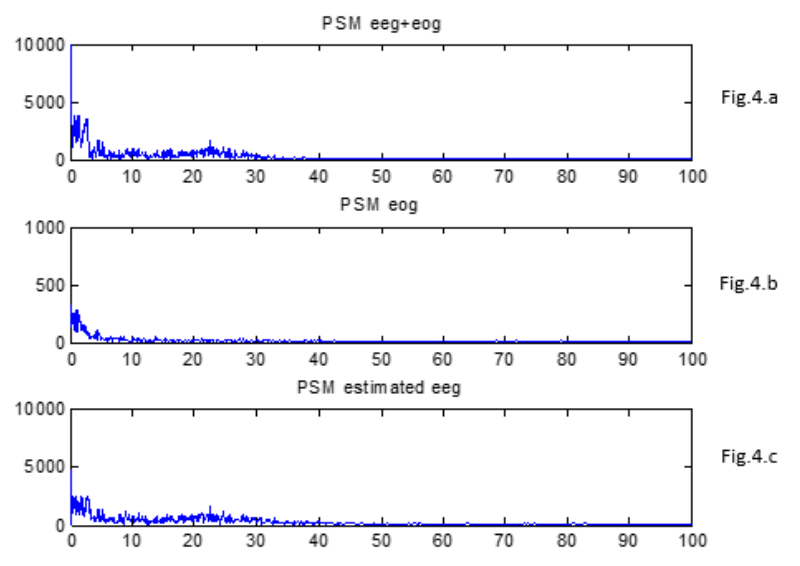

Figure 4. power spectrum of signals

\section{Discussion and Conclusion}

An adaptive noise cancellation technique was studied and used to de-noise life signals. Simulation results show that the proposed adaptive RBFN-based filter can cancel the noise signal from the distorted information signal successfully.

In this research, the results of method were discussed and analyzed.

\section{References}

[1] R. Jonathan, J. M. Dennis, B. Niels, P. Gert and M. Theresa, "Brain computer interfaces for communication and control," pp.767-791, Clinical Neurophysiology, 2002.

[2] M. Fatourechi, A. Bashashati, K. W. Rahab and E. B. Gary, "EMG and EOG artifacts from BCI systems: a Survey," pp. 480-494, Clinical Neurophysiology, 2007.

[3] P.He, M.Kahle, G.Wilson and C.Russel, "Removal of ocular artifacts from EEG: A comparison of Adaptive filtering method and regression method using simulated data", IEEE engineering in medicine and Biology 27th annual conference, 2005 .

[4] Ehsan Zezhadaraya, Mohammed B Shamosollahi. " EOG artifact removal from EEG using ICA and ARMAX modeling", Private communication, 2004. 
24 Mohammad Seifi et al: : Adaptive Noise Cancellation for Eliminating Artifacts of Life Signals Using Fuzzy and Neural Networks

[5] P. He, G.Wilson and C.Russel, "Removal of Ocular artifacts from Electro encephalogram by adaptive Filtering," pp.407412, Medical \&Biological Engineering \& Computing, 2004.

[6] B.W.Jervis, M.Thomlinson, C.Mair, J.M. Lopez and M.I.B Garcia, "Residual ocular artifacts subsequent to ocular artifact removal from the electroencephalogram", pp.293-298, IEE Proceedings-Volume 146, Issue 6, Nov. 1999.

[7] T. D. Lagerlund, F. W. Sharbrough, and N. E. Busacker, "Spatial filtering of multichannel electroencephalographic recordings through principal component analysis by singular value decomposition," J. Clin. Neurophysiol., vol. 14, pp. 7382, 1997.
[8] R. Verleger, T. Gasser, \& J. Möcks, "Correction of EOG artifacts in eventrelated potentials of the EEG: Aspects of reliability and validity", Psychophysiology, vol. 19, pp 472480, 1982.

[9] J. L. Whitton, F. Lue, \& H. Moldofsky, "A spectral method for removing eye movement artifacts from the EEG", Electroenceph. Clin. Neurophysiol. vol. 44, pp. 735-741, 1978

[10] J.S.R. Jaug, "Anfis: Adaptivenetwork-based fuzzy inference system," IEEE Trans. on Systems,Man,and Cybernetics, vol. 23, pp. 665-685, 1993.

[11] www.physionet.org 